\title{
SUBOPTIMALITY IN LINEAR CONTROL SYSTEMS WITH TIME DELAY
}

\author{
W. L. CHAN
}

(Received 24 August 1980)

\begin{abstract}
For the linear quadratic control problem with delay, a lower bound for the performance index is obtained by elementary methods. Using this bound, two important $a$ posteriori error estimates are derived. The first one measures the deviation of the performance index while the second is for the deviation of the state and control variables from the optimal solution.
\end{abstract}

\section{Introduction}

We consider a linear controlled system modelled by the delay-differential equation

$$
\dot{Y}(t)=A(t) Y(t)+C(t) Y(t-r)+B(t) U(t),
$$

with $Y(t)$ an $n$-vector, $U(t)$ an $m$-vector, $A(t), B(t)$ and $C(t)$ continuous matrices on $[0, T]$. Further, $U(t) \in L_{2}[0, T]$, the space of square integrable functions. The continuous initial data is specified by $Y(t)=h(t)$ for $-r \leqslant t \leqslant 0$. The cost functional of control is

$$
J_{1}(U)=\frac{1}{2} \int_{0}^{T}\left(Y^{\prime}(t) Q(t) Y(t)+U^{\prime}(t) R(t) U(t)\right) d t
$$

where $Q(t)=Q^{\prime}(t)>0$ and $R(t)=R^{\prime}(t)>0$ are positive-definite matrices of appropriate dimensions; $r$ and $T$ are positive real numbers $T>r$. This problem has been extensively studied by Chyung and Lee [7] (see also [1]) who proved that

(c) Copyright Australian Mathematical Society 1982 
the minimization of $J_{1}(U)$, subject to (1), is equivalent to solving the following two point boundary value problem with both delayed and advanced arguments:

$$
\begin{aligned}
\dot{y}(t) & =A(t) y(t)+C(t) y(t-r)-B(t) R^{-1}(t) B^{\prime}(t) p(t), \\
-\dot{p}(t) & =A^{\prime}(t) p(t)+C^{\prime}(t+r) p(t+r)+Q(t) y(t), \\
y(t) & =h(t) \text { for }-r \leqslant t \leqslant 0 \quad \text { and } p(t)=0 \text { for } t>T .
\end{aligned}
$$

The optimal controller is then given by

$$
u(t)=-R^{-1}(t) B^{\prime}(t) p(t) .
$$

Existence and uniqueness of absolutely continuous optimal solutions $y(t)$ and $p(t)$ were also proved in [7]. For any admissible control $U(t)$ in $L_{2}[0, T]$, by definition of the optimal control $u(t)$,

$$
J^{*} \equiv J_{1}(u(t)) \leqslant J_{1}(U(t)) .
$$

Our problem at hand is to establish a lower bound $J_{2}(U(t))$, where $U(t)$ belongs to a certain subset of $L_{2}[0, T]$, with the property

$$
J_{2}(U(t)) \leqslant J_{2}(u(t))=J^{*},
$$

and more importantly, to deduce from it computable a posteriori estimates for the following non-negative suboptimality measures defined by

$$
\begin{aligned}
& \text { (i) } d_{J}=J_{1}(U)-J^{*}, \\
& \text { (ii) } d_{Y}=\int_{0}^{T}\|Y(t)-y(t)\|_{Q(t)}^{2} d t, \\
& \text { (iii) } d_{U}=\int_{0}^{T}\|U(t)-u(t)\|_{R(t)}^{2} d t,
\end{aligned}
$$

where $\|Y(t)\|_{Q}^{2}$ denotes $Y^{\prime}(t) Q(t) Y(t)$ and $Y(t)$ is the state generated by the control $U(t)$ while $y(t), u(t)$ and $p(t)$ are the optimal state and costate, that is, solutions to (1.3)-(1.6).

A lower bound for the cost functional in variational control problems with time delay was first discussed by Chan [3], [4]. For the no-delay case, one can consult [9], [6], [2], [5] and the references therein. The results about $d_{J}, d_{Y}$ and $d_{U}$, in the context of a posteriori suboptimal estimates are new. Notice that $d_{Y}^{1 / 2}$ is an equivalent $L_{2}$ norm between $Y$ and $y$ and similarly $d^{1 / 2}$ is an equivalent $L_{2}$ norm between $U$ and $u$.

\section{Preliminaries}

From the form of the optimal controller (1.6), we are interested in considering the set of admissible controls generated by

$$
U(t)=-R^{-1}(t) B^{\prime}(t) W(t)
$$


where $W(t)$ is an arbitrary $n$-vector in $L_{2}[0, T]$. For such a control, $U(t)$, the state associated with it, is thus

$$
\begin{aligned}
& \dot{Y}(t)=A(t) Y(t)+C(t) Y(t-r)-M(t) W(t), \\
& Y(t)=h(t) \text { for }-r \leqslant t \leqslant 0,
\end{aligned}
$$

where $M(t)=B(t) R^{-1} B^{\prime}(t)$ and the corresponding costate is given by

$$
\begin{aligned}
-\dot{P}(t) & =A^{\prime}(t) P(t)+C^{\prime}(t+r) P(t+r)+Q(t) Y(t), \\
P(t) & =0 \quad \text { for } t \geqslant T .
\end{aligned}
$$

In what follows, suboptimal bounds on $d_{J}, d_{Y}$ and $d_{U}$ will be given in terms of $W(t)$ and $P(t)$ alone.

Alternatively, we may consider the question of assigning a meaningful 'cost' to any $n$-vector $\bar{Y}(t) \in L_{2}[0, T]$, which may be, say, arrived at by some approximating scheme to the state equation (1.1). To this end, for such a given $\bar{Y}(t)$, we consider the function $\bar{P}(t)$ satisfying

$$
\begin{aligned}
-\dot{\bar{P}}(t) & =A^{\prime}(t) \bar{P}(t)+C^{\prime}(t+r) \bar{P}(t+r)+Q(t) \bar{Y}(t), \\
\bar{P}(t) & =0 \quad \text { for } t \geqslant T,
\end{aligned}
$$

and $Z(t)$ given by

$$
\begin{aligned}
& \dot{Z}(t)=A(t) Z(t)+C(t) Z(t-r)-M(t) \bar{P}(t), \\
& Z(t)=h(t) \text { for }-r \leqslant t \leqslant 0 .
\end{aligned}
$$

Suboptimal bounds on $d_{\left.J_{(} \bar{Y}\right)}, d_{\bar{Y}}$ and $d_{\bar{P}}$, defined analogously to $d_{J}, d_{Y}$ and $d_{U}$, will be given in terms of $\bar{Y}(t)$ and $Z(t)$. We could interpret (2.1)-(2.5) as a kind of 'forward-backward sweep' while (2.6)-(2.9) form a 'backward-forward sweep'.

LEMMA 1. The optimal cost functional $J(u(t))$ can be expressed as

$$
\begin{aligned}
J= & p^{\prime}(0) h(0)-\frac{1}{2} \int_{0}^{T}\left[\|p(t)\|_{M(t)}^{2}+\|y(t)\|_{Q(t)}^{2}\right] d t \\
& +\int_{-r}^{0} p^{\prime}(t+r) C(t+r) h(t) d t .
\end{aligned}
$$

Proof. Multiply (1.3) by $p^{\prime}(t)$ and the transpose of (1.4) by $y(t)$. Integrating the difference from 0 to $T$ gives

$$
\begin{aligned}
\left.p^{\prime}(t) y(t)\right|_{0} ^{T}= & -\int_{0}^{T}\left[\|p(t)\|_{M(t)}^{2}+\|y(t)\|_{Q(t)}^{2}\right] d t \\
& +\int_{0}^{T}\left[p^{\prime}(t) C(t) y(t-r)\right] d t-\int_{0}^{T-r}\left[p^{\prime}(L+r) C(t+r) y(t)\right] d t \\
= & -\int_{0}^{T}\left[\|p(t)\|_{M(t)}^{2}+\|y(t)\|_{Q(t)}^{2}\right] d t+\int_{0}^{r}\left[p^{\prime}(t) C(t) y(t-r)\right] d t
\end{aligned}
$$


Hence, using (1.5), and continuity of $p$,

$$
p^{\prime}(0) h(0)=2 J-\int_{-r}^{0}\left[p^{\prime}(t+r) C(t+r) h(t)\right] d t,
$$

from which the result follows.

LEMMA 2. If $X, Y, Z$ are n-vectors and $Q$ is an $n \times n$ symmetric matrix, then

$$
\|X-Z\|_{Q}^{2}-\|Y-Z\|_{Q}^{2}=\|X-Y\|_{Q}^{2}+2(X-Y)^{\prime} Q(Y-Z) .
$$

\section{Main result}

THEOREM 1. Assumptions and notations are as in (2.1)-(2.5). If

$$
J_{1}(W)=\frac{1}{2} \int_{0}^{T}\left(\|Y(t)\|_{Q(t)}^{2}+\|W(t)\|_{M(t)}^{2}\right) d t
$$

and

$$
\begin{gathered}
J_{2}(W)-P^{\prime \prime}(0) h(0)-\frac{1}{2} \int_{0}^{T}\left(\|Y(t)\|_{Q(t)}^{2}+\|P(t)\|_{M(t)}^{2}\right) d t \\
+\int_{-r}^{0} P^{\prime}(t+r) C(t+r) h(t) d t,
\end{gathered}
$$

then

$$
\begin{gathered}
\text { (a) } J_{2}(W) \leqslant J \leqslant J_{1}(W) \quad \text { and } \\
\text { (b) } J_{1}(W)-J_{2}(W)=\frac{1}{2} \int_{0}^{T}\|W(t)-P(t)\|_{M}^{2} d t .
\end{gathered}
$$

Proof. (a)

$$
\begin{aligned}
J-J_{2}(W)= & {[p(0)-P(0)]^{\prime} h(0) } \\
& +\frac{1}{2} \int_{0}^{T}\left[\|Y(t)\|_{Q(t)}^{2}-\|y(t)\|_{Q(t)}^{2}+\|P(t)\|_{M(t)}^{2}-\|p(t)\|_{M(t)}^{2}\right] d t \\
& -\int_{-r}^{0}[P(t+r)-p(t+r)]^{\prime} C(t+r) h(t) d t .
\end{aligned}
$$

Using Lemma 2 in the first integral, we find

$$
\begin{aligned}
J-J_{2}(W)= & {[p(0)-P(0)]^{\prime} h(0) } \\
& +\int_{0}^{T}\left[(Y(t)-y(t))^{\prime} Q y(t)+(P(t)-p(t))^{\prime} M(t) p(t)\right] d t \\
& +\frac{1}{2} \int_{0}^{T}\left[\|Y(t)-y(t)\|_{Q(t)}^{2}+\|P(t)-p(t)\|_{M(t)}^{2}\right] d t \\
& -\int_{-r}^{0}[P(t+r)-p(t+r)]^{\prime} C(t+r) h(t) d t
\end{aligned}
$$


Now, by (1.3),

$$
\begin{aligned}
\int_{0}^{T} & (P(t)-p(t))^{\prime} M(t) p(t) d t \\
= & \int_{0}^{T}(P(t)-p(t))^{\prime}(-\dot{y}(t)+A(t) y(t)+C(t) y(t-r)) d t \\
= & \int_{0}^{T}(\dot{P}(t)-\dot{p}(t))^{\prime} y(t)+(P(t)-p(t))^{\prime}(A(t) y(t)+C(t) y(t-r)) d t \\
& -\left.(P(t)-p(t))^{\prime} y(t)\right|_{0} ^{T} \\
= & \int_{0}^{T}\left[-(Y(t)-y(t))^{\prime} Q(t) y(t)\right] d t \\
& +\int_{-r}^{0}[P(t+r)-p(t+r)]^{\prime} C(t+r) y(t) d t \\
& +\left(P^{\prime}(0)-p^{\prime}(0)\right) h(0)
\end{aligned}
$$

where the last equality follows from (1.4), (1.5), (2.4) and (2.5). Substituting (3.6) into (3.5) gives

$$
\begin{aligned}
J-J_{2}(W) & =\frac{1}{2} \int_{0}^{T}\left[\|Y(t)-y(t)\|_{Q(t)}^{2}+\|P(t)-p(t)\|_{M(t)}^{2}\right] d t \\
& =\frac{1}{2} \int_{0}^{T}\left[\|Y(t)-y(t)\|_{Q(t)}^{2}+\|U(t)-u(t)\|_{R(t)}\right] d t
\end{aligned}
$$

so that $J_{2}(W) \leqslant J$. The other inequality, $J \leqslant J_{1}(W)$, follows from the definition of $J$.

(b) Equations (2.2)-(2.5) imply

$$
\frac{d}{d t}\left[P^{\prime}(t) Y(t)\right]=\left\{\begin{array}{r}
-\left[P^{\prime}(t) M(t) W(t)+\|Y(t)\|_{Q(t)}^{2}\right]+P^{\prime}(t) C(t) Y(t-r) \\
-P^{\prime}(t+r) C(t+r) Y(t) \text { for } 0 \leqslant t \leqslant T-r, \\
-\left[P^{\prime}(t) M(t) W(t)+\|Y(t)\|_{Q(t)}^{2}\right]+P^{\prime}(t) C(t) Y(t-r) \\
\text { for } T-r<t \leqslant T .
\end{array}\right.
$$

Integrating the above from 0 to $T$ yields,

$$
\begin{aligned}
P^{\prime}(0) h(0)= & \int_{0}^{T}\left[P^{\prime}(t) M(t) W(t)+\|Y(t)\|_{Q(t)}^{2}\right] d t \\
& -\int_{-r}^{0} P^{\prime}(t+r) C(t+r) h(t) d t
\end{aligned}
$$

Putting (3.8) into (3.2) results in

$$
J_{2}(W)=\frac{1}{2} \int_{0}^{T}\left[\|Y(t)\|_{Q(t)}^{2}-\|P(t)\|_{M(t)}^{2}+2 P^{\prime}(t) M(t) W(t)\right] d t
$$


and therefore with (3.1)

$$
\begin{aligned}
J_{1}(W)-J_{2}(W) & =\frac{1}{2} \int_{0}^{T}\left[\|W(t)\|_{M(t)}^{2}-2 P^{\prime}(t) M(t) W(t)+\|P(t)\|_{M(t)}^{2}\right] d t \\
& =\frac{1}{2} \int_{0}^{T}\|P(t)-W(t)\|_{M(t)}^{2} d t \geqslant 0 .
\end{aligned}
$$

COROLlaRy 1. With the assumptions and notations in Theorem 1,

$$
\text { (i) } d_{J} \leqslant \frac{1}{2} \int_{0}^{T}\|W(t)-P(t)\|_{M(t)}^{2} d t
$$

and

$$
\text { (ii) } d_{Y}+d_{U} \leqslant \int_{0}^{T}\|W(t)-P(t)\|_{M(t)}^{2} d t \text {. }
$$

Proof. Since $J_{2}(W) \leqslant J \leqslant J_{1}(W)$ from Theorem 1(a), it follows that

$$
d_{J}(W)=J_{1}(W)-J \leqslant J_{1}(W)-J_{2}(W)=\frac{1}{2} \int_{0}^{T}\|W(t)-P(t)\|_{M(t)}^{2} d t .
$$

Also, from Theorem 1(b),

$$
\frac{1}{2}\left(d_{Y}+d_{U}\right)=J-J_{2}(W) \leqslant J_{1}(W)-J_{2}(W)=\frac{1}{2} \int_{0}^{T}\|W(t)-P(t)\|_{M(t)}^{2} d t .
$$

Now if we take the 'backward-forward sweep' view point, that is considering (2.6)-(2.9), we have

THEOREM 2. Given an arbitrary $n$-vector $\bar{Y}(t)$ in $L_{2}[0, T]$, and if

$$
J_{1}(\bar{Y})=\frac{1}{2} \int_{0}^{T}\left[\|Z(t)\|_{Q(t)}^{2}+\|\bar{P}(t)\|_{M(t)}^{2}\right] d t
$$

and

$$
\begin{aligned}
J_{2}(\bar{Y})= & -\frac{1}{2} \int_{0}^{T}\left[\|\bar{Y}(t)\|_{Q(t)}^{2}+\|\bar{P}(t)\|_{M(t)}^{2}\right] d t \\
& +\bar{P}(0) h(0)+\int_{-r}^{0} \bar{P}(t+r) C(t+r) h(t) d t
\end{aligned}
$$

then

$$
\begin{aligned}
& \text { (a) } J_{2}(\bar{Y}) \leqslant J \leqslant J_{1}(\bar{Y}) \quad \text { and } \\
& \text { (b) } J_{1}(\bar{Y})-J_{2}(\bar{Y})=\int_{0}^{T}\|Z(t)-\bar{Y}(t)\|_{Q(t)}^{2} d t \text {. }
\end{aligned}
$$

This theorem leads to bounds for $d_{J}(\bar{Y})$ and $d_{\bar{Y}}+d_{\bar{P}}$ in Corollary 2 . 
COROLlary 2. With the assumptions and notations in Theorem 2,

$$
\text { (i) } d_{J}(\bar{Y}) \equiv J_{1}(\bar{Y})-J \leqslant \frac{1}{2} \int_{0}^{T}\|Z(t)-\bar{Y}(t)\|_{Q(t)}^{2} d t \text {, }
$$

and

$$
\text { (ii) } \begin{aligned}
d_{\bar{Y}}+d_{\bar{P}} & \equiv \int_{0}^{T}\left[\|\bar{Y}(t)-y(t)\|_{Q(t)}^{2}+\|\bar{P}(t)-p(t)\|_{M(t)}^{2}\right] d t \\
& \leqslant \int_{0}^{T}\|Z(t)-\bar{Y}(t)\|_{Q(t)}^{2} d t .
\end{aligned}
$$

The proofs of Theorem 2 and Corollary 2 are similar to those of Theorem 1 and Corollary 1 , and hence are omitted. Instead, we give an example in the following section for illustration.

\section{An example}

Consider the example given in [8]:

$$
\operatorname{Min} J_{1}=\frac{1}{2} \int_{0}^{2}\left(y^{2}+u^{2}\right) d t
$$

subject to

$$
\begin{aligned}
& \dot{y}=y(t)+y(t-1)+u(t) \quad \text { for } 0 \leqslant t \leqslant 2, \\
& y(t)=1 \quad \text { for }-1 \leqslant t \leqslant 0 .
\end{aligned}
$$

Our aim here is to demonstrate the implications of Theorem 2. The costate equation of (4.2) is

$$
\begin{aligned}
-\dot{p}(t) & =\left\{\begin{array}{l}
p(t)+y(t) \text { for } 1 \leqslant t \leqslant 2, \\
p(t)+y(t)+p(t+1) \text { for } 0 \leqslant t \leqslant 1,
\end{array}\right\} \\
p(2) & =0
\end{aligned}
$$

and the optimal control is given by the solutions of (4.2), (4.3) with $u(t)=-p(t)$. Suppose we have chosen $\bar{Y}$ in (3.12) of Theorem 2 as

$$
\bar{Y}(t)=\left\{\begin{array}{lr}
1, & -1 \leqslant t \leqslant 0 \\
1-13 t+(-a+2) t^{2}, & 0 \leqslant t \leqslant 1 \\
(a-8)-(a+3) t-(a-5) t^{2}, & 1 \leqslant t \leqslant 2
\end{array}\right\} .
$$

Then, the solution to (4.3) (compare with (2.6)), with $\bar{Y}(t)$ taking the place of $y(t)$, can be shown to be

$$
-\bar{P}(t)=\left\{\begin{array}{ll}
(a-2)+(-2-a) t+t^{2}, & 0 \leqslant t \leqslant 1 \\
5+(a-13) t+(-a+5) t^{2}, & 1 \leqslant t \leqslant 2
\end{array}\right\},
$$

where $a=-0.5$. 
Now $Z(t)$ of $(2.8)$, needed for the evaluation of $J_{1}(\bar{Y})$ in (3.12), can also be seen to be

$$
Z(t)=\left\{\begin{array}{lr}
1, & -1 \leqslant t \leqslant 0 \\
1+a t-t^{2}, & 0 \leqslant t \leqslant 1 \\
-1+5 t+(a-4) t^{2}, & 1 \leqslant t \leqslant 2
\end{array}\right\} .
$$

With $\bar{Y}(t), \bar{P}(t)$ and $Z(t)$ at hand, $J_{1}$ and $J_{2}$ are then calculated according to (3.12) and (3.13) respectively, giving

$$
\begin{aligned}
& J_{1}=18.8958333, \\
& J_{2}=-11.7345238
\end{aligned}
$$

and, from [8], the optimal value of $J_{1}$ is

$$
J^{*}=6.1701 \text {. }
$$

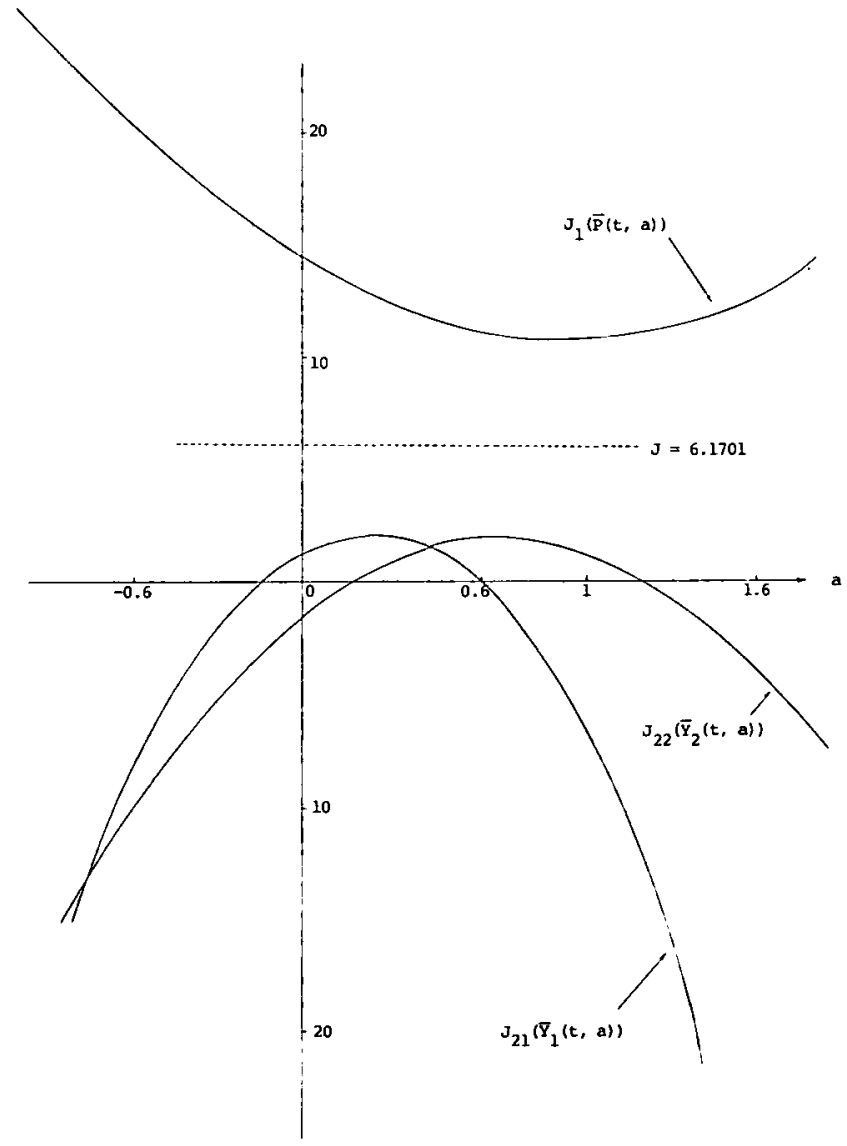

Figure 1. Suboptimality from $J_{1}$ and $J_{2}$. 
Thus, indeed, $J_{2} \leqslant J^{*} \leqslant J_{1}$ but the large difference, $J_{1}-J_{2}=30.6303571$, indicates there is much room for improvement. In fact if we consider $a$ in (4.5) as a parameter and define $U(t)=-\bar{P}(t, a)$ then $Z(t, a)$ in (4.6) gives a family of states and the corresponding $J_{1}(a)$ has been plotted in Figure 1. To apply Theorem 2 to estimate how far away are these families of controls and states from the optimal solution, we arbitrarily choose two families of functions defined by

$$
\bar{Y}_{1}(t, a)=\left\{\begin{array}{lr}
1, & -1 \leqslant t \leqslant 0 \\
1+(2.1-7 a) t+(-3.1+6 a) t^{2}, & 0 \leqslant t \leqslant 1 \\
-0.7+(0.7-2 a) a t^{2}, & 1 \leqslant t \leqslant 2
\end{array}\right\}
$$

and

$$
\bar{Y}_{2}(t, a)=\left\{\begin{array}{lr}
1, & -1 \leqslant t \leqslant 0 \\
1+(3 a-1.4) t+(0.2-3 a) t^{2}, & 0 \leqslant t \leqslant 1 \\
-a+(a-0.4) t+0.2 t^{2}, & 0 \leqslant t \leqslant 2
\end{array}\right\} .
$$

Substituting these into (2.6) (compare with (4.3)) and solving for $\bar{P}(t)$, gives

$$
-P_{1}(t, a)=\left\{\begin{array}{ll}
(-5.2+9 a)+(7.6-13 a) t+(-3.1+5 a) t^{2}, & 0 \leqslant t \leqslant 1 \\
(-1.4+4 a)+(0.7-4 a) t+a t^{2}, & 1 \leqslant t \leqslant 2
\end{array}\right\} .
$$

and

$$
-P_{2}(t, a)=\left\{\begin{array}{ll}
(0.8-6 a)+(-0.6+8 a) t-3 a t^{2}, & 0 \leqslant t \leqslant 1 \\
(0.8-2 a)+(a-0.8) t+a t^{2}, & 1 \leqslant t \leqslant 2
\end{array}\right\} .
$$

Using (4.7) and (4.9), $J_{21}(a)$ in (3.13) is evaluated. Similarly (4.8) and (4.10) are used to evaluate $J_{22}(a)$, (that is, $J_{1}(a)$ and $J_{2 i}(a)$ using the functions $\bar{Y}_{1}$ and $\bar{Y}_{2}$ respectively). They are plotted against $a$ in Figure 1. The 'gap' between $\min _{a} J_{1}(a)$ and $\max _{a, i=1,2}\left\{J_{2 i}(a)\right\}$, gives the required estimate of suboptimality of the control $U(t)=-P(t, a)$, as in (4.5), applied to (4.2).

\section{References}

[1] Y. Alekal, P. Brunovsky, D. H. Chyung and E. B. Lee, "The quadratic problem for systems with time delay", IEEE Trans. Automat. Contr. AC-16(1971), 673-687.

[2] E. Aronoff and C. T. Leondes, "Lower bounds for a quadratic cost functional", Int. J. Systems Sci. 7 (1976), 17-25.

[3] W. L. Chan, "Dual variational principles with delayed argument", Int. J. Contr. 20 (1974), 1015-1019. 
[4] W. L. Chan, "Variational dualities in the linear regulator and estimation problems with and without time delay", J. Inst. Maths. Applics. 18 (1976), 237-247.

[5] W. L. Chan, "A posteriori suboptimal error estimates in control problems", Appl. Math. and Computation 3 (1977), 291-306.

[6] W. L. Chan, G. G. Leininger and J. B. Farison, "Complementary variational principle and duality in mathematical programming", J. Math. Anal. Appl. 43 (1973), 348-356.

[7] D. H. Chyung and E. G. Lee, "Linear optimal systems with time delays", SIAM J. Contr. 4 (1966), 548-575.

[8] D. H. Eller, J. K. Aggarwal and H. T. Banks, "Optimal control of linear time-delay systems", IEEE Trans. Automat. Contr. AC-14 (1969), 678-687.

[9] J. D. Pearson, "Reciprocity and duality in control programming problems", J. Math. Anal. Appl. 10 (1965), 388-408.

\section{Department of Mathematics}

The Chinese University of Hong Kong

Shatin, N. T.

Hong Kong 